\title{
La observancia del pluralismo político en el audio- visual mediterráneo: autoridades independientes y criterios de regulación de la televisión
}

\author{
Ricardo CARNIEL BugS \\ Universidad Autónoma de Barcelona \\ ricardo.carniel@uab.es \\ Fernando SABÉs TURMo \\ Universidad Autónoma de Barcelona \\ fernando.sabes@uab.es
}

\section{Resumen:}

El fortalecimiento de la democracia es uno de los objetivos destacados por las políticas de integración entre Europa y países del sur del Mediterráneo. Los medios audiovisuales tienen un papel fundamental, si reflejan en sus contenidos las diversas corrientes políticas de una sociedad, por lo que la regulación de este aspecto es esencial. Este artículo analiza las autoridades reguladoras del audiovisual en Francia, Marruecos y las experiencias autonómicas de Andalucía y Cataluña. Los cuatro reguladores tienen funciones relacionadas principalmente con los contenidos informativos, por lo que identificaremos qué criterios utilizan y qué actuaciones realizaron entre 2009 y 2011 respecto su misión de velar por el pluralismo político en la TV.

Palabras clave: Regulación de contenidos; Pluralismo; Televisión; Mediterráneo

The observance of political pluralism in Mediterranean media: independent authorities and regulatory criteria for television

\begin{abstract}
:
Strengthening democracy is one of the main objectives of the policies of integration between Europe and Southern Mediterranean countries. Audiovisual media have a key role if its contents reflect the various political tendencies of a society, so the regulation of this aspect is essential. This paper analyzes the regulatory authorities in France, Morocco and the experiences of Spanish autonomous communities of Andalusia and Catalonia. The four regulators have functions mainly related news content. We aim to identify what criteria they use and what actions were performed between 2009 and 2011 regarding their mission of ensuring political pluralism on TV.
\end{abstract}

Key Words: Content regulation; Pluralism; Television; Mediterranean

\section{Referencia normalizada:}

Carniel Bugs, R. y Sabés Turmo, F. (2014): La observancia del pluralismo político en el audiovisual mediterráneo: autoridades independientes y criterios de regulación de la televisión. Historia y Comunicación Social. Vol. 19. Núm. Especial Febrero. Págs. 273-286.

\section{Sumario:}

1. Introducción. 2. Autoridades independientes y la regulación del audiovisual. 3. Criterios y seguimiento del pluralismo político en la TV. 3.1. Francia. 3.2. Marruecos. 3.3. Cataluña y Andalucía. 4. Conclusiones. 5. Referencias. 


\section{Introducción}

Las iniciativas de acercamiento y colaboración con países del sur del Mediterráneo han sido, históricamente, un elemento clave en la política exterior de Europa. A finales de 1995, la primera Conferencia Euromediterránea de Ministros de Asuntos Exteriores en Barcelona marcó el comienzo de unas relaciones multilaterales avanzadas, a través del Partenariado o Asociación Euromediterránea ${ }^{1}$. En 2008, los Estados miembros decidieron darle un nuevo impulso relanzando el proceso como Unión por el Mediterráneo, pero manteniendo y reforzando valores y preceptos reflejados en sus documentos desde sus inicios, tales como los derechos fundamentales y la dignidad humana, la creación de una zona de libre comercio, el diálogo intercultural y el fortalecimiento de la democracia (UpM, 2008), siendo este último el que particularmente nos ocupa.

La consolidación de sistemas democráticos en la región es un objetivo reconocido y respaldado por otras iniciativas paralelas como la Política Europea de Vecindad o la Alianza de Civilizaciones de la ONU, que también contribuyen a promover un espacio común entre ambas orillas mediterráneas.

En este sentido, además de informar a la opinión pública sobre las acciones desarrolladas, los medios de comunicación tienen un rol destacado para la consecución de muchos objetivos. Para el fortalecimiento de la democracia, los medios deberían favorecer el pluralismo, reflejando las diferentes corrientes políticas e ideologías presentes en una sociedad (Klimkiewicz, 2010).

En el sector audiovisual, el concepto de pluralismo está frecuentemente asociado a la diversidad de medios (pluralismo externo) a partir del fin de monopolios estatales y normas anti-concentración de la propiedad, pero también atañe al pluralismo interno, esto es, el pluralismo en los contenidos. Del conjunto de aspectos asociados al pluralismo en el audiovisual (religioso, social, de género, etc.), nos interesamos especialmente por el pluralismo político en la televisión. Pero, ¿de qué forma se puede garantizar la observancia del pluralismo en la TV? Aquí cobran especial protagonismo las autoridades encargadas de regular el sector.

Con el foco sobre el Mediterráneo, investigaremos los organismos reguladores del audiovisual en Francia, Marruecos y - dada la inexistencia de un ente independiente estatal en España - de las autoridades autonómicas de Cataluña y Andalucía. Siguiendo el análisis de base documental, verificaremos si ejecutan funciones relacionadas con el pluralismo político en las televisiones bajo su competencia, así como si dichas autoridades comparten criterios en el sentido de armonizar los marcos regulatorios en la cuenca mediterránea ${ }^{2}$. 


\section{Autoridades independienets y la regulación del audiovisual}

La efectividad de una regulación independiente exige que la autoridad responsable esté dotada de un alto nivel de autonomía respecto los poderes político y económico. Por ello, la creación de reguladores independientes también está asociada a la democratización de las sociedades, en la medida en que contribuye a alejar el poder político del control directo sobre el sector audiovisual. Asimismo, dichos organismos tienen el reto de asegurar el equilibrio entre los intereses del mercado y de la ciudadanía (Lunt y Livingstone, 2012). En este epígrafe, verificaremos si los organismos analizados fueron legalmente instados a vigilar la observancia del pluralismo político en la televisión.

Francia fue uno de los pioneros a nivel global. El Conseil Supérieur de l'Audiovisuel (CSA) fue constituido en 1989, aunque sus bases empezaron a sentarse en 1982, con la Ley sobre la comunicación audiovisual, y en 1986, con Ley relativa a la libertad de comunicación ${ }^{3}$. Ambos textos y sus posteriores actualizaciones son los pilares de la normativa audiovisual francesa, que establece el Principio de Pluralismo como un objetivo constitucional. Así, el CSA recibió la misión de velar por el respeto de la expresión plural de corrientes de pensamiento y de opinión en la radio y la televisión, especialmente en las emisiones de información política y general ${ }^{4}$.

Entre sus funciones específicas, el Consejo debe fijar los criterios de garantía del pluralismo político en la televisión e informar periódicamente al Parlamento sobre la presencia e intervención de los representantes políticos en los contenidos, a partir de un seguimiento continuo de los programas. El CSA también define las reglas para la producción y emisión de la campaña política en los medios de gestión pública y fiscaliza su aplicación. Otra función asociada al pluralismo político es la de revisar y fijar para el año siguiente qué organizaciones políticas, sindicales o profesionales de representación nacional pueden acceder a lo que en Francia se conoce como el tiempo "d'expression directe", esto es, espacios en la programación de la TV pública para que dichas agrupaciones difundan sus ideas y acciones.

El CSA también ha inspirado a otros países como Marruecos que, en 2002, reformó su legislación audiovisual, empezando por establecer una nueva autoridad reguladora. La Haute Autorité de la Communication Audiovisuelle (HACA) fue instituida por un Real Decreto previo al fin del monopolio estatal de televisión ${ }^{5}$. Como en el caso francés, la norma no otorgó personalidad jurídica al organismo. Pero, al contrario del CSA, la ley marroquí no presentaba ninguna mención expresa a la independencia del regulador 6 . Bajo una "tutela del Rey", la HACA fue encargada de varias funciones, entre ellas la de velar por el pluralismo de opiniones y pensamiento en medios públicos y privados, especialmente en la información política ${ }^{7}$. Posteriormente, nuevos cambios legislativos resultaron en la Loi $n^{\circ} 77-03$ relative à la communication audio$v_{\text {visuelle }}^{8}$, en vigor desde 2005, para regular emisiones privadas y públicas. Tanto el texto que creó la HACA como la Ley de Comunicación Audiovisual de Marruecos presentan una evidente inspiración en la legislación francesa, por lo que plasman ideales como la consagración de la libertad de comunicación, la garantía de las liber- 
tades de expresión, de opinión y comunicación individual y colectiva, el pluralismo de las corrientes de pensamiento y los principios democráticos. En este sentido, la potestad reglamentaria de la HACA le otorga competencia para determinar y supervisar las reglas relativas al pluralismo político.

Pese a la cercanía con ambos países anteriores, España sigue siendo un caso anómalo, ya que no cuenta con una autoridad independiente de ámbito estatal. En 2010, la nueva Ley General de Comunicación Audiovisual actualizó la normativa del sector y establecía el Consejo Estatal de Medios Audiovisuales (CEMA) ${ }^{9}$ que, hasta la actualidad, no ha salido del papel. Pero la nueva Ley General refrendó la misión de garantizar el pluralismo político, social y cultural en los contenido ${ }^{10} \mathrm{y}$ el papel de los consejos audiovisuales creados por las Comunidades Autónomas.

Cataluña fue la pionera, cuando en 1996 instauró el Consell de l'Audiovisual de Catalunya (CAC), creado inicialmente como órgano asesor del Gobierno catalán. En el año 2000 una reforma legislativa otorgó al CAC un estatus diferenciado. La llamada Ley del $\mathrm{CAC}^{11}$ reconvirtió el Consell en un ente público con personalidad jurídica propia y autonomía funcional.

Esta configuración legal trajo nuevos compromisos y potestades, similares al modelo francés, incluida la función de velar por el pluralismo interno y externo de los medios audiovisuales. Posteriormente, la nueva Llei de la Comunicació Audiovisual de Catalunya reforzó esta misión, estableciendo el pluralismo como condición esencial para el cumplimiento de las libertades de expresión, información y comunicación ${ }^{12}$. Esta ley y sus actualizaciones también definen el pluralismo como un elemento clave de la misión de servicio público atribuida a los medios, siendo el CAC ratificado como la autoridad responsable por vigilar la pluralidad de medios y debe presentar periódicamente informes sobre el nivel de pluralismo político en la televisión.

El segundo ejemplo autonómico es el Consejo Audiovisual de Andalucía (CAA), creado en 2004 como una entidad pública con personalidad jurídica propia y plena autonomía ${ }^{13}$. Aunque el Gobierno andaluz también se inspiró en los modelos existentes en Europa y el Mediterráneo, el encargo de funciones y el traspaso de poderes fue más limitado. Comparado con sus homólogos de Francia, Marruecos y Cataluña, el CAA no tiene potestad para actuar en los concursos públicos de adjudicación de frecuencias (pluralismo externo), que sigue en manos del Gobierno.

Sobre el pluralismo interno, el regulador andaluz debe velar por el cumplimiento de los principios democráticos, una comunicación libre y el pluralismo político, social, religioso, cultural ${ }^{14}$. No obstante, como no tiene potestad reglamentaria, tampoco puede desarrollar las normas y criterios objetivos de observancia del pluralismo.

Así, podemos constatar que las cuatro autoridades reguladoras incluidas en el estudio tienen competencias sobre los contenidos audiovisuales, aunque las funciones relacionadas con el pluralismo político y los mecanismos disponibles son diferentes. 


\section{Criterios y seguimiento del pluralismo político en la tv}

El pluralismo es un principio ampliamente reconocido y su dimensión interna (Valcke, 2012) constituye un factor clave para fortalecer la democracia. En este epígrafe, buscaremos identificar qué criterios utilizan los reguladores para supervisar los contenidos televisivos y qué actuaciones reguladoras relacionadas con el pluralismo político en la televisión fueron llevadas a cabo entre 2009 y 2011. Nuestra base documental está formada principalmente por los informes anuales de actividades de los reguladores, además de otros recursos como publicaciones en Diario Oficial o documentos facilitados por las propias autoridades en sus páginas web.

Una característica común a todos los casos es el refuerzo de la supervisión en períodos cercanos a las elecciones. Esto es, la regulación se ejerce de forma continua, pero con especificidades entre los períodos electorales - que incluyen las campañas y comicios - y el resto del tiempo. Por otro lado, se detecta una serie de aspectos concretos que pasamos a analizar.

\subsection{Francia}

En el primer año de nuestro análisis, 2009, cuatro votaciones concentraron la atención del CSA francés: tres comicios regionales y la elección de representantes al Parlamento Europeo. El Consejo emitió recomendaciones específicas para los procesos regionales, organizó los tiempos de campaña en los medios públicos y evaluó la cobertura política. No constan incidencias relevantes y el CSA consideró que las fuerzas políticas recibieron un trato equitativo. Sobre las elecciones europeas las tareas fueron similares, a partir de la elaboración de un documento ${ }^{15}$ con reglas simplificadas y tiempo de aplicación reducido para "aligerar" las restricciones sobre la libertad de los medios (CSA, 2010: 107). En general, el Consejo valoró positivamente el tiempo otorgado a los partidos y la cobertura de la información política. Ninguna de las 20 quejas recibidas resultó en sanción. Dos emisoras fueron advertidas por difundir sondeos de opinión en períodos no autorizados. Y el canal público France 3 recibió un requerimiento por el trato desigual de políticos de un mismo partido durante sus elecciones primarias.

La supervisión en períodos no electorales dio origen a informes mensuales que ya venían siendo realizados en años anteriores, pero con un cambio importante en el segundo semestre de 2009. El CSA acordó reformar el Principio de pluralismo político, en vigor desde el año 2000, que seguía la regla de tres tercios del tiempo repartidos entre partidos de situación, oposición y sin representación parlamentaria. A partir de un dictamen del Consejo de Estado sobre las intervenciones del Presidente de la República en los medios, el regulador adoptó un nuevo cálculo. En resumen, el nuevo principio garantiza un mínimo del 50\% del tiempo total a la oposición y, según el tema abordado, incluye o no las intervenciones del Presidente de la República en el cómputo del bloque "mayoritario"16. 
Por último, también le corresponde al regulador autorizar los tiempos d'expression directe. Este año, el CSA fijó un calendario de emisiones para seis formaciones políticas y 13 organizaciones sindicales o profesionales ${ }^{17}$.

En 2010, el CSA constató que los operadores de TV habían aplicado satisfactoriamente el nuevo principio de pluralismo. Como cada año, se publicaron los informes sobre la presencia de las fuerzas políticas en informativos, magacines y otros programas. Asimismo, el Consejo analizó las quejas recibidas, como la de un diputado que denunciaba una "denigración sistemática" del Presidente de la República en programas de entretenimiento. Los miembros del CSA entendieron que los contenidos indicados estaban "dentro de los límites del derecho al humor y la caricatura, reconocido por la jurisprudencia" (CSA, 2011: 102), pero recordaron a los medios su responsabilidad editorial en posibles casos de difamación o vulneración de la privacidad. La emisora TF1 fue advertida por no incluir la versión de las autoridades locales y de la policía en una noticia sobre inseguridad en un municipio francés. También en 2010 se realizaron elecciones para la Asamblea de Córcega y dos consultas públicas en Martinica y Guayana. Para cada votación el Consejo adoptó orientaciones específicas sobre el tratamiento informativo de las campañas y de la difusión de resultados, además de organizar la campaña oficial. En las tres ocasiones, el regulador evaluó positivamente la equidad de los tiempos dedicados a los partidos. Un año más, el CSA revisó y determinó el sistema de emisiones d'expression directe.

Ya en 2011, el pluralismo político volvió a acaparar la atención del regulador francés debido a los preparativos para las presidenciales de 2012. Se adoptó una nueva Resolución general, revisando los criterios a seguir por los medios en períodos electorales, además de regular la obligación de incluir subtitulación o lenguaje de signos en los programas relacionados con los $\operatorname{comicios}^{18}$. También se publicó una Recomendación relativa a las elecciones cantonales. Ambos textos tienen como principio básico la equidad en la cobertura informativa que, además, debe ser rigurosa y honesta. Paralelamente, el organismo regulador realizó dos audiencias con los partidos políticos para explicar el marco jurídico.

Pese a todo, el CSA identificó durante los meses de junio, julio y agosto graves desequilibrios en el tiempo de palabra concedido a la oposición parlamentaria en tres emisoras (BFM TV, i>Télé, LCI), que recibieron requerimientos. A finales de año, el Consejo publicó una Deliberación, que estableció los períodos de campaña electoral en los medios, las condiciones específicas para la cobertura de los candidatos, hasta la divulgación de resultados de los comicios. En la organización de la campaña oficial en los medios públicos, el CSA aseguró que la propaganda electoral debería estar subtitulada y audiodescrita, para la accesibilidad de personas con limitaciones visuales y auditivas.

\subsection{Marruecos}

Al contrario del CSA francés, en el período considerado la HACA no tenía la obligación legal de publicar informes de actividades. Así, hemos analizado las decisiones 
publicadas en el Boletín Oficial de Marruecos y los documentos disponibles en el sitio oficial del regulador marroquí.

Para períodos no electorales, la HACA también tiene una regla general de proporcionalidad ${ }^{19}$. Además de fijar criterios para sindicatos y organizaciones sociales, la normativa determina que los medios deben velar para que el tiempo de intervención del Gobierno y de partidos de mayoría parlamentaria no sea superior al doble del dedicado a la oposición. Asimismo, se establece un tiempo mínimo para las ideas de partidos sin representación parlamentaria.

Periódicamente, los operadores audiovisuales han de enviar a la HACA sus informes sobre pluralismo en telediarios, debates y otros programas informativos, a través de los cuales el regulador fiscaliza el cumplimiento de las normas. Así, en 2009 la HACA publicó cuatro informes trimestrales sobre el pluralismo en los telediarios, dos estudios semestrales sobre el pluralismo en magacines informativos y otras emisiones, y otro informe específico sobre el pluralismo durante el período de las elecciones municipales. La cadena pública SNRT recibió una notificación por incumplir los criterios de pluralismo. Otras dos quejas fueron interpuestas por cinco organizaciones sindicales, que denunciaban los dos principales canales de TV (Al Oula y 2M) de promover un "apagón informativo" sobre una huelga nacional. La HACA consideró que 2M había cumplido sus obligaciones, al contrario de Al Oula que emitió únicamente la posición del Gobierno. Poco tiempo después, la HACA volvió a llamar la atención de $\mathrm{Al}$ Oula por negar la palabra a un partido político. Los criterios de pluralismo político fueron reiterados cuando también este año la HACA aprobó los nuevos cahiers des charges de los tres operadores estatales, SNRT, SOREAD-2M y MEDI 1 SAT.

En 2010, la HACA adoptó un nuevo formato para los informes de seguimiento de la TV, que pasaron a observar también el tiempo de palabra ocupado por mujeres, la lengua utilizada (árabe, francés, amazigh) y la representación territorial, a través de portavoces regionales. Fueron publicados dos informes trimestrales sobre telediarios y un informe semestral sobre otros programas informativos. Los tres abarcan el primer semestre de 2010, pero no consta si llegaron a producirse los mismos informes en el segundo semestre.

$\mathrm{Al}$ año siguiente, el paisaje mediático y sociopolítico tuvo cambios. La llamada Primavera Árabe de países vecinos no llegó a reproducirse con la misma intensidad en Marruecos, aunque también trajo repercusiones como el proyecto de una nueva Constitución, sometido a un referéndum popular. Antes de la votación, la HACA hizo recomendaciones específicas para la garantía de libertad de expresión y pluralismo de opiniones. Asimismo, supervisó la programación televisiva para observar el cumplimiento de dichos principios hasta la realización del referéndum. Para el regulador los resultados fueron positivos, con equilibrio en la participación de actores políticos, institucionales, sociales y otros ${ }^{20}$.

Posteriormente, el país empezó otro período de campaña para las elecciones legislativas. La HACA publicó una Decisión, reforzando los criterios de proporcionalidad 
para el pluralismo político y las normas relativas a la honestidad y neutralidad de la información durante los comicios y el recuento de $\operatorname{votos}^{21}$. Un nuevo informe demostró, por un lado, resultados satisfactorios en el pluralismo político, aunque, por otro, una inmensa disparidad en la cuestión de género (más del 90\% del tiempo de palabra ocupado por hombres). El regulador también lamentó el escaso esfuerzo de los operadores en incluir subtitulación o lengua de signos en los contenidos políticos para las personas con discapacidad auditiva ${ }^{22}$.

\subsection{Cataluña y Andalucía}

En el caso español, la ausencia de un regulador estatal independiente es la paradoja de dos reconocidas autoridades de ámbito autonómico, los Consejos Audiovisuales de Cataluña (CAC) y de Andalucía (CAA), siendo el pluralismo un aspecto relevante para ambos.

Así, en 2009 las elecciones al Parlamento Europeo merecieron especial atención por parte de los reguladores. El CAC realizó un informe y constató que ningún operador catalán, excepto el ente público, dio espacio a las agrupaciones políticas sin representación parlamentaria. También se verificó que el único canal privado (8tv) había realizado una escasa cobertura de la campaña, con evidente desequilibrio entre partidos. Fuera del período electoral, el CAC hizo el seguimiento del pluralismo político en la TV, publicando informes mensuales sobre la materia. A diferencia de los casos anteriores, en Cataluña no hay un principio de pluralismo legalmente definido, esto es, la observancia del pluralismo se ampara en una relativa "proporcionalidad" de las fuerzas políticas y criterios generales de equidad, profesionalidad e interés informativo ${ }^{23}$. Paralelamente, el CAC introdujo la cuestión de género, elaborando un estudio sobre la presencia femenina en las noticias y en la información política.

Ya en Andalucía, el CAA empezó a publicar en 2009 los primeros análisis sobre el pluralismo político en las televisiones públicas andaluzas. El Consejo divulgó cuatro estudios trimestrales sobre el pluralismo en los telediarios del ente público RTVA, de las desconexiones de la TV estatal RTVE, y de diversos operadores locales de titularidad municipal. Los resultados generaron también el primer Informe Anual sobre el Pluralismo Político, con datos relevantes sobre la presencia y el tiempo de palabra de las administraciones y de los partidos, así como de otros actores sociales como los sindicatos ${ }^{24}$. El CAA también abordó la igualdad de género, una de sus líneas prioritarias de actuación, por lo que analizó el reparto del tiempo de palabra entre hombres y mujeres.

En 2010, el Consejo andaluz realizó actuaciones diferenciadas. Además de la publicación de los estudios trimestrales y del Informe anual sobre pluralismo político, el CAA instó a una emisora local (Ejido TV) a cesar inmediatamente la emisión de publicidad política y le advirtió de posibles sanciones. Asimismo, un informe específico analizó el tratamiento dado por los medios a la decisión del Tribunal Supremo, que desestimó una querella del Partido Popular contra el Presidente del Gobierno autonómico ${ }^{25}$. 
En Cataluña, el CAC publicó un año más los informes mensuales de la programación regular y un informe específico sobre la información durante la campaña al Parlamento autonómico. Los resultados fueron similares a comicios anteriores: un alto nivel de cumplimiento en la TV pública, que tiene este requisito en su misión de servicio público, y notables desequilibrios en medios privados, que no cuentan con una normativa concreta sobre la equidad de fuerzas políticas ${ }^{26}$.

El último año analizado, 2011, tuvo doble cita electoral en ambas comunidades autónomas: elecciones municipales y las generales del Estado español, convocadas de forma anticipada.

En Cataluña, el CAC impulsó un grupo de trabajo entre representantes de partidos políticos, medios, profesionales de la comunicación y de la investigación, del que resultaron propuestas ${ }^{27}$ de un sistema más flexible sin renunciar a la normativa electoral vigente (CAC, 2012: 20). Posteriormente, el CAC monitorizó ambas campañas electorales y los comicios, con resultados similares: un mayor compromiso de los medios públicos que los privados, tanto en el volumen de información como en la observancia del pluralismo. Fuera de los períodos electorales, el CAC envió al Parlamento los informes mensuales de pluralismo político en los programas informativos.

En Andalucía, los comicios motivaron un incremento de trabajo para el Consejo Audiovisual que recibió 74 denuncias relativas al pluralismo político y falta de neutralidad en la información, especialmente sobre medios de gestión municipal (CAA, 2012: 37). Del total de quejas, fueron emitidas siete resoluciones y una decisión dando la razón a los reclamantes por irregularidades en los canales Fuengirola TV, Torrevisión, Ejido TV y Macarena TV. Todos fueron advertidos sobre las obligaciones del servicio público audiovisual. También se enviaron requerimientos de cese inmediato de emisión a cuatro operadores que difundían publicidad política ilegal, además de trasladar todos los casos a la Junta Electoral. Como en años anteriores, fueron realizados cuatro estudios trimestrales sobre el pluralismo político en las televisiones públicas de Andalucía, así como el Informe Anual del pluralismo. El Consejo emitió también un documento de valoración de los estudios, en el que manifestó su particular preocupación con las emisoras municipales, muchas de las cuales no conceden espacios significativos a los partidos de oposición, predominando la presencia de políticos gobernantes ${ }^{28}$. La inexistencia de reglas claras limita la intervención del CAA. Desde hace años el Consejo demanda al Parlamento andaluz que reconozca legalmente un principio claro de medición del pluralismo político, con el cual el regulador podría actuar de forma más contundente en esta materia.

\section{Conclusiones}

Alineados con las principales políticas de integración euromediterránea, los organismos reguladores de Francia, Marruecos, Cataluña y Andalucía sí tienen el pluralismo como un principio clave en la supervisión de los medios audiovisuales. A partir 
de las actuaciones analizadas, identificamos que los cuatro casos tienen competencias para monitorizar los canales de TV y solicitar a los operadores el envío de datos o copias de la programación para verificar el cumplimiento de las reglas. En todos los casos los reguladores tienen encargadas una serie de funciones, entre las cuales está la de velar por una comunicación libre y plural, reflejando diversos ámbitos de pluralismo como el religioso, el lingüístico, el cultural y, particularmente, el pluralismo político de cada contexto. Así, los organismos reguladores analizados cometen sus tareas, verificando los niveles de observancia del pluralismo político en la TV.

Entre 2009 y 2011, la actuación más constante fue la publicación de informes sistemáticos, que verifican si los medios respetan reglas de pluralismo político. Los criterios más frecuentes son: el tiempo de noticia, la presencia y el tiempo de palabra concedido a las fuerzas políticas. Estos informes funcionan como instrumento de presión tanto de los propios partidos interesados como por parte de la audiencia, condicionando la imagen de las emisoras que no abren espacio a las diversas corrientes ideológicas. También es un elemento común la inclusión de la variable de género en los estudios, evidenciando, aunque en diferentes grados, el espacio minoritario ocupado por las mujeres en la vida pública.

Aunque el modelo francés haya sido una referencia para las otras autoridades, la regulación del pluralismo presenta diferencias sustanciales. Empezando por la existencia de una regla clara de pluralismo político. El CSA francés tiene la potestad para establecer el llamado Principio de Pluralismo, con el que contabiliza el equilibrio de tiempo entre las fuerzas políticas del país. La HACA de Marruecos también cuenta con esta potestad, desarrollando las reglas de pluralismo, pero su sistema no es tan generoso con los partidos de oposición como en Francia. En Cataluña, aunque tenga potestad reglamentaria, el CAC no ha llegado a concretar reglas específicas de pluralismo, limitándose a publicar acuerdos con carácter de recomendación. La observancia del pluralismo se basa en criterios generales de equidad y proporcionalidad de la representación política derivada de las votaciones. Ya en Andalucía, el CAA no tiene potestad reglamentaria, por lo que lleva años pidiendo al Parlamento autonómico que pacte un principio claro de pluralismo, mientras trabaja con recomendaciones y normas generales relacionadas.

La existencia de criterios claros de pluralismo, reconocidos legalmente, es un factor determinante para respaldar los informes publicados, así como para permitir actuaciones más contundentes en el caso de incumplimientos graves y reiterados. Francia y Marruecos cuentan con unas reglas específicas y de fácil verificación, por lo que sus reguladores llegaron a emitir requerimientos - paso previo a la sanción - a diferentes televisiones. En el caso catalán, no constan este tipo de actuaciones durante el período analizado, siendo que los informes del CAC indicaron niveles aceptables de pluralismo. Ya en Andalucía, los desequilibrios entre fuerzas políticas en la televisión son un problema crónico, particularmente entre las emisoras gestionadas por ayuntamientos. La falta de un criterio consensuado impide que el regulador tome medidas más severas. 
A modo de consideraciones finales, entendemos que los organismos independientes de regulación tienen un rol decisivo en la supervisión del pluralismo en televisión y en el fomento de los valores democráticos. El modelo francés de regulación es una referencia para los países mediterráneos, pero existen notables diferencias tanto en la configuración de la autoridad reguladora como en los criterios de pluralismo en la televisión, siendo el caso de Marruecos una norma más favorable al poder político. El caso de España es paradigmático, ya que las iniciativas de ámbito autonómico ponen de manifiesto las lagunas derivadas de la ausencia de un regulador estatal. Así, los países mediterráneos comparten unos principios comunes hacia el fortalecimiento de la democracia, pero necesitan dar varios pasos para alcanzar una verdadera política reguladora común, que favorezca la integración regional.

\section{Bibliografía}

CARNIEL BUGS, R. (2013). Los organismos reguladores del audiovisual y sus actuaciones en la zona euromediterránea. La protección de los menores, los derechos humanos y la diversidad cultural en Francia, España, Líbano y Marruecos. Tesis doctoral. Bellaterra (Cerdanyola del Vallès): Universitat Autònoma de Barcelona.

CONSEIL SUPÉRIEUR DE L'AUDIOVISUEL (2010): Rapport Annuel 2009. París:

CSA, Service de l'information et de la documentation.

(2011). Rapport Annuel 2010. París: CSA, Service de l'information et de la documentation.

(2012). Rapport Annuel 2011. París: CSA, Service de l'information et de la documentation.

CONSEJO AUDIOVISUAL DE ANDALUCÍA (2010): Memoria 4. Informe 2009 del Consejo Audiovisual de Andalucía al Parlamento de Andalucía. Sevilla: CAA, Junta de Andalucía.

(2011): Memoria 5. Informe 2010 del Consejo Audiovisual de Andalucía al Parlamento de Andalucía. Sevilla: CAA, Junta de Andalucía.

(2012). Memoria 6. Informe 2011 del Consejo Audiovisual de Andalucía al Parlamento de Andalucía. Sevilla: CAA, Junta de Andalucía.

CONSELL DE L'AUDIOVISUAL DE CATALUNYA (2010). Memòria Anual 2009.

Barcelona: CAC.

(2011). Memòria Anual 2010. CAC. Barcelona.

(2012). Memòria Anual 2011. CAC. Barcelona.

KLIMKIEWICZ, B. (ed.) (2010). Media Freedom and Pluralism. Budapest - Nueva York: CEU.

LUNT, P. y LIVINGSTONE, S. (2012). Media regulation: governance and the interests of citizens and consumers. Londres: Sage.

UNION POR EL MEDITERRANEO (2008). Final Statement. Marseille, 3-4 November 2008. Recuperado día 04 de diciembre de 2012 de http://www.ufmsecretariat. org/en/wp-content/uploads/2010/12/dec-final-Marseille-UfM.pdf 
VALCKE, P. (2012). "Desafíos de la regulación del pluralismo en los medios en la Unión Europea: el potencial de la regulación basada en el riesgo". En Quaderns del $C A C, \mathrm{n}^{\circ} 38$, vol. XV (1). Junio. Barcelona: CAC. p. 25-36. Recuperado día 10 de enero de 2013 de: http://www.cac.cat/pfw_files/cma/recerca/quaderns_cac/ Q38_valcke_ES.pdf

\section{Notas}

1 Declaración de Barcelona, firmada por los entonces 15 miembros de las Comunidades Europeas y 12 socios: Argelia, Chipre, Egipto, Israel, Jordania, Líbano, Malta, Marruecos, Autoridad Nacional Palestina, Siria, Túnez y Turquía (Barcelona, 27-28 de noviembre de 1995).

2 Este artículo contiene resultados de la tesis doctoral de Carniel Bugs (2013) titulada "Los organismos reguladores del audiovisual y sus actuaciones en la zona euromediterránea. La protección de los menores, los derechos humanos y la diversidad cultural en Francia, España, Líbano y Marruecos", que fue desarrollada en el marco del proyecto "La construcción social del espacio euromediterráneo en los medios de comunicación. La información en prensa y televisión" (CSO2008-01579), financiado por el Ministerio español Ciencia e Innovación (2009-2011), y en el que participaran como investigadores los autores de este texto. Los temas estudiados tienen seguimiento actualmente con el proyecto "La construcción social del espacio intermediterráneo y sus correlaciones en la agenda temática de los medios de comunicación. La información en prensa y televisión" (CSO2012-35955), financiado por el Ministerio español de Economía y Competitividad (2013-2016). Investigadores por orden alfabético: Hesham Abu-Sharar, M. Luz Barbeito, Ricardo Carniel Bugs, Carmina Crusafon, Olga Del Río, Lucrecia Escudero, Natalia Fernández, Elisabet García, Juan Antonio G. Galindo, Víctor Gutiérrez, Nouredinne Harrami, Carmen Martínez, Patria Román, Fernando Sabés, Susana Tovías, José Juan Verón y Teresa Velázquez (Investigadora Principal). Doctorandos adscritos: Hicham El Bakhouti, Estíbaliz Ortega y Valentina Saini. Colaboradores (pendientes de incorporación administrativa): Zhou Bouzidi y Carolina Cerdá-Guzmán.

3 Loi n'82-652 sur la communication audiovisuelle y Loi $n^{\circ}$ 86-1067 relative à la liberté de communication, publicadas en el Journal Officiel de la République Française (JORF) de 30/07/1982 y de 01/10/1986, respectivamente. La Ley del 82 instituía la Haute Autorité de la communication audiovisuelle, sustituida en 1986 por la Commission nationale de la communication et des libertés. Tres años después, la Ley del 86 fue modificada (Loi n89-25, du 17 janvier 1989, modifiant la Loi 86-1067, JORF de 18/01/1989) y el CSA pasa a ser definitivamente el regulador del audiovisual francés, definido como autoridad independiente, aunque no tenga la personalidad jurídica propia (personnalité morale).

4 Artículo 13, Loi 86-1067.

5 Dahir $n^{\circ}$ 1-02-212 portant création de la Haute Autorité de la communication audiovisuelle. Publicado en el Bulletin Officiel du Royaume du Maroc (BORM) núm. 5036, de 05/09/2002.

6 A mediados de 2011, la aprobación por referéndum popular de la nueva Constitución de Marruecos incluye posibilidades de cambio para la HACA. El Título XII trata de la buena gobernanza y hace mención a las instituciones dedicadas a la regulación, atribuyéndoles la independencia en el ejercicio de sus funciones (artículo 159). La mención a la independencia debería permitir cambios relevantes como la elaboración propia de su reglamento interno.

7 Artículo 3.8 del Dahir $n^{\circ}$ 1-02-212. 
8 Dahir $n^{\circ} 1-04-257$, de 07/01/2005, portant promulgation de la loi $n^{\circ} 77-03$ relative à la communication audiovisuelle. BORM $\mathrm{n}^{\circ} 5288$, de 03/02/2005.

9 Ley 7/2010, de 31 de marzo, General de la Comunicación Audiovisual. BOE núm. 79, de 01/04/2010.

10 Artículo 4.5 de la Ley $7 / 2010$.

11 Llei 2/2000, de 4 de maig, del Consell de l'Audiovisual de Catalunya, publicada en el Diari Oficial de la Generalitat de Catalunya (DOGC) núm. 3133, de 05/05/2000.

12 Artículo 5 de la Llei 22/2005, de 29 de desembre, de la comunicació audiovisual de Catalunya. DOGC núm. 4543, de 03/01/2006.

13 Ley 1/2004, de 17 de diciembre. Boletín Oficial de la Junta de Andalucía (BOJA) núm. 254, de $30 / 12 / 2004$.

14 Artículo 4 de la Ley 1/2004.

15 Recommandation du 24 mars 2009 en vue de l'élection des représentants au Parlement européen le 7 juin 2009, modificada por la Recommandation du 21 avril 2009 y la Recommandation du 5 mai 2009. Disponibles en: http://www.csa.fr/Espace-juridique/Deliberations-et-recommandations-du-CSA/ Recommandations-du-CSA-en-vue-de-consultations-electorales-ou-referendaires/Recommandation-du-5-mai-2009-modifiant-la-recommandation-du-24-mars-2009-en-vue-de-1-election-des-representants-au-Parlement-europeen-le-7-juin-2009 [12/01/2012].

16 Délibération du 21 juillet 2009 relative au principe de pluralisme politique dans les services de radio et de télévision. Disponible en: http:/www.csa.fr/Espace-juridique/Deliberations-et-recommandations-du-CSA/Recommandations-et-deliberations-du-CSA-relatives-a-d-autres-sujets/Deliberation-du-21-juillet-2009-relative-au-principe-de-pluralisme-politique-dans-les-services-de-radio-et-de-television [12/01/2012].

17 Décision no 2009-795 du 8 décembre 200. JORF de 17/12/2009.

18 Délibération du 4 janvier 2011 relative au principe de pluralisme politique dans les services de radio et de télévision en période électorale. Disponible en: http://www.csa.fr/Television/Le-suivi-des-programmes/Le-pluralisme-politique-et-les-campagnes-electorales/Deliberation-du-4-janvier-2011-relative-au-principe-de-pluralisme-politique-dans-les-services-de-radio-et-de-television-en-periode-electorale [04/09/2012].

19 Décision du CSCA $N^{\circ}$ 46-06 du 27 Septembre 2006 relative aux règles de la garantie du pluralisme d'expression des courants de pensée et d'opinion dans les services de communication audiovisuelle en dehors de périodes électorales. Disponible en: http://www.haca.ma/html/pdf/46-06.pdf [01/04/2012]. Asimismo, la HACA publicó en 2007 reglas específicas para el período de elecciones generales.

20 Rapport final relatif au respect de l'expression pluraliste des courants de pensée et d'opinion dans les médias audiovisuels pendant la période de consultation référendaire de 2011. Disponible en: http://www.haca.ma/pdf/HACA_ref2011_full_def2.pdf [11/09/2012].

21 Décision du CSCA $n^{\circ} 46-11$ relative a la garantie du pluralisme politique pendant la période des élections législatives générales (2011) dans les médias audiovisuels. Disponible en: http://www.haca. $\mathrm{ma} / \mathrm{html} / \mathrm{pdf} /$ Decision\%20CSCA\%204611\%20legislatives\%202011_VF.pdf [12/09/2012].

22 Rapport de suivi de la période électorale 2011 dans les médias audiovisuels marocains. Disponible en: http://www.haca.ma/pdf/Rapport\%20final\%20legislatives\%202011.pdf [20/09/2012].

23 Acord 78/2007, de 7 de Març, del CAC. Disponible en: http://www.cac.cat/web/actuacions/index. jsp?Njk\%3D\&MQ\%3D\%3D\&L3dlYi9hY3R1YWNpb25zL2xsaXN0YXRDb250ZW50\&NQ\%$3 \mathrm{D} \% 3 \mathrm{D} \#[23 / 01 / 2012]$. 
24 Informe Anual Pluralismo Político 2009. Disponible en: http://www.consejoaudiovisualdeandalucia. es/sites/default/files/publicaciones/informe_anual_pluralismo_politico_2009.pdf [29/03/2012].

25 Informe 03/2010, ampliación del informe 14/2009 sobre el tratamiento informativo del caso Matsa. Disponible en: http://www.consejoaudiovisualdeandalucia.es/sites/default/files/informes/ Informe_2010_03_Ampliacion\%20Matsa.pdf [22/07/2012].

26 Informe especific de pluralisme a la televisió i a la ràdio durant la campanya de les eleccions al Parlament de Catalunya 2010 (del 12 al 26 de novembre). Valoració del Consell de l'Audiovisual de Catalunya (aprovada en la sessió de l'1 de desembre de 2010). Disponible en: http://www.cac.cat/ $\mathrm{pfw}$ files/cma/actuacions/Continguts/Valoracio_pluralisme_campanya_2010.pdf [29/07/2012].

27 Document sobre la qualitat de la informació en les campanyes electorals. Disponible en: http:// www.cac.cat/web/actuacions/index.jsp?MjE\%3D\&MQ\%3D\%3D\&L3dlYi9hY3R1YWNpb25zL2xsaXN0YXRDb250ZW50\# [04/09/2012].

28 Documento de análisis y valoración sobre el Informe de pluralismo político en las televisiones públicas de Andalucía. Disponible en: http://www.consejoaudiovisualdeandalucia.es/sites/default/ files/publicacion/pdf/1205/6_3_2_propuesta_decision_informe_anual_2011.pdf [11/09/2012].

\section{Los autores}

Ricardo Carniel Bugs. Doctor en Comunicación y Periodismo por la Universidad Autónoma de Barcelona y periodista. Es profesor investigador postdoctoral en el departamento de Periodismo y Ciencias de la Comunicación de la Facultad de Ciencias de la Comunicación de la UAB. Es miembro del Laboratori de Prospectiva i Recerca en Comunicació, Cultura i Cooperació (LAPREC) y del Observatorio Mediterráneo de la Comunicación (OMEC) de la UAB. Ha publicado artículos y capítulos de libros sobre estructura y políticas de comunicación, con énfasis en la regulación de los medios audiovisuales.

Fernando Sabés Turmo. Doctor en Comunicación Audiovisual y Publicidad por la Universidad Autónoma de Barcelona. Es profesor titular en el departamento de Periodismo y Ciencias de la Comunicación de la Facultad de Ciencias de la Comunicación de la Universidad Autónoma de Barcelona, donde ha sido vicedecano y actualmente es vicedirector de docencia del mencionado departamento. Ha escrito diferentes libros entre los que destacan los elaborados junto a José Juan Verón Lassa: La eficacia de lo sencillo. Introducción a la práctica del periodismo (2008) y La gestión de la información en la administración local (2008). También ha escrito numerosos artículos en revistas científicas del ámbito de la comunicación y varios capítulos de libros. Ha participado en diferentes proyectos de investigación del ámbito de la comunicación financiados a través del Plan Nacional. 